\title{
Uso de distintos tipos de fibras para mejorar las propiedades mecánicas de la mezcla asfáltica: Una revisión literaria
}

\section{Use of different types of fibers to improve the mechanical properties of asphalt: A literary review}

\section{Orlando Jefferson Adrianzen Flores}

Escuela Profesional de Ingeniería Civil

Universidad Señor de Sipán

Chiclayo, Perú

afloresorlandoj@crece.uss.edu.pe

\section{Jhon Jeiner Azula Vasquez}

Escuela Profesional de Ingeniería Civi

Universidad Señor de Sipán

Chiclayo, Perú

avasquezjhonjei@crece.uss.edu.pe

\author{
Ernesto Dante Rodríguez Lafitte \\ Docente, Escuela Profesional de Ingeniería Civil \\ Universidad Señor de Sipán \\ Chiclayo, Perú \\ rlafitte@crece.uss.edu.pe
}

\author{
Sócrates Pedro Muñoz Pérez \\ Director, Escuela Profesional de Ingeniería Civil \\ Universidad Señor de Sipán \\ Chiclayo, Perú \\ msocrates@crece.uss.edu.pe
}

\section{Cristian Fabian Pacherres Sánchez}

Escuela Profesional de Ingeniería Civil

Universidad Señor de Sipán

Chiclayo, Perú

psanchezcris@crece.uss.edu.pe

Fecha de recepción: 29/07/2021 - Fecha de aprobación: 30/11/2021

\section{RESUMEN}

La demanda del uso de pavimentos flexibles va creciendo raudamente debido a su buen desempeño, pero este está siendo afectado de manera considerable debido al aumento del tráfico y las variaciones climáticas, esto trae consigo la presencia de fallas considerables en el pavimento, causando que su desempeño disminuya y, por ende, se acorte su tiempo de vida útil. Este documento tiene como objetivo realizar una revisión sistemática de los distintos tipos de fibras usados en las mezclas asfálticas para la mejora de sus propiedades mecánicas, además de estudiar los porcentajes y longitudes óptimas, las pruebas utilizadas, el desempeño de las mezclas según el tipo de betún, el tipo de mezcla asfáltica y, finalmente, se revisa la rentabilidad que proporciona el uso de fibras en las mezclas asfálticas. Se revisaron 81 artículos publicados en las bases de datos de Scopus y Ebsco, entre los años 2014 al 2021, los cuales tuvieron el mismo objetivo de estudiar las características mecánicas de las mezclas asfálticas con adición de fibras. Se concluyó que las fibras, además de mejorar las propiedades de los pavimentos flexibles, son rentablemente buenas ya que, a pesar de tener un aumento en el costo inicial, demuestran un incremento en el ciclo de vida, siendo así económicamente factibles para proyectos viales que se realicen con el uso mezclas asfálticas, además se recomendó proseguir con estudios encaminados a utilizar este tipo de mezclas asfálticas.

Palabras clave: fibras, propiedades mecánicas, mezcla asfáltica, desempeño del cemento asfáltico

\section{ABSTRACT}

The demand for the use of flexible pavements is growing rapidly due to its good performance, but this is being affected considerably due to increased traffic and climatic variations, this brings with it the presence of considerable failures in the pavement, causing its performance to decrease and, therefore, its useful lifetime is shortened. This document aims to carry out a systematic review of the different types of fibers used in asphalt mixtures to improve their mechanical properties, in addition to studying the optimal percentages and lengths, the tests used, the performance of the mixtures according to the type of bitumen, the type of asphalt mix, and finally the profitability provided by the use of fibers in asphalt mixes is reviewed. Eightyone articles published between 2014 and 2021 in the Scopus and Ebsco databases were reviewed. The reviewed papers had the same objective of studying the mechanical characteristics of asphalt mixtures using fibers. It was concluded that the fibers improve the properties of flexible pavements and, in addition, are profitably good despite having an increase in the initial cost, they demonstrate an increase in the life cycle, thus being economically feasible for road projects that are carried out with the use flexible pavements. It is also recommended to continue with studies aimed at using this type of asphalt mixture.

Keywords: fibers, mechanical properties, asphalt mixture, asphalt cement performance 


\section{Introducción}

El pavimento asfáltico es usado extensamente alrededor del mundo debido a su buen rendimiento, resaltando principalmente su fácil proceso constructivo y su valor relativamente bajo, este tipo de pavimento está constituido en su mayoría por una mezcla asfáltica (Guo, Li, Cheng, Jiao, y Xu, 2015). Esta mezcla bituminosa está conformada en esencia por la unión de cemento asfáltico, áridos y relleno, siendo el cemento asfáltico en conjunto con el relleno quienes conforman la mezcla asfáltica (Musa, Shanbara, y Dulaimi, 2020), pero esta se estropea al pasar el tiempo, como consecuencia de la combinación de diversos factores medioambientales y la incidencia de carga generada por los medios de transporte pesados (Eisa, Basiouny, y Daloob, 2021). Es así que hasta las carreteras de superficie bituminosa bien diseñada se están estropeando de manera acelerada, afectando su desempeño en la transitabilidad y así reduciendo su vida útil (Kar, Nagabhushana, y Jain, 2019). También influye de manera notable en su duración el uso de insumos de mala calidad en el diseño y producción de la mezcla, si bien es cierto en el mercado se presentan insumos de buena calidad, pero su adquisición suele tornarse difícil debido, en esencia, a su elevado costo monetario (Klinsky Gutiérrez, Kaloush, Faria, y Dos Santos Bardini, 2018). Es así que, debido a estas variables que afectan al pavimento, en unos pocos años se presentan signos de fallas estructurales en la superficie, fallas que para su reparación, se tiene que valer de un consumo energético adicional al que se ha utilizado en primera instancia para su construcción, aumentando la emisión de gases de efecto invernadero $y$, con esto, las repercusiones negativas para la salud de los seres vivos (Guan et al., 2019).

Dentro de las fallas más recurrentes está la formación de surcos, originados por la tensión aglomerada en el cemento asfáltico, la forma y el entrelazado de los áridos. Esta falla apresura el deterioro de los pavimentos, incrementando los costos generales de construcción, referente al valor de mantenimiento y reparaciones (Al-Sabaeei et al., 2020). Por otro lado, está el agrietamiento por fatiga que ocurre debido al incremento de micro y macro grietas provocadas por el peso vehicular, viéndose influenciada también por la combinación de cambios en la temperatura, humedad y envejecimiento. Esta falla se manifiesta como una de las de mayores dificultades, causantes de la reducción del tiempo de vida útil de la mezcla asfáltica (Usman, Masirin, Ahmad, y Ali, 2019). Si bien es cierto se puede reparar el daño causado al aplicarse una capa de mezcla, este tratamiento suele tornarse algo ineficiente ya que el desempeño mejorará solo por un corto tiempo, esto debido a que las imperfecciones del pavimento anterior se reflejarán raudamente en la superficie, producto de los efectos mezclados de las tensiones incitadas térmicamente y del peso del tránsito (Romeo, Freddi, y Montepara, 2014). En busca de minimizar las fallas, se propone optimizar las características mecánicas de la mezcla asfáltica mediante la utilización de distintos tipos de fibra, que se acentúan como materiales novedosos en la mezcla debido a su rentabilidad (Saleem y Ismael, 2020).

Es así que, con miras de mejorar el desempeño y aumentar el tiempo de vida de un pavimento, y así presentar una mejor calidad de servicio, diversos investigadores han utilizado aditivos para mejorar las características de la mezcla asfáltica, aditivos que refieren a diversos tipos de fibras (Mohammed, Parry, Thom, y Grenfell, 2020). Dentro de las diversas fibras sintéticas utilizadas, se usaron macrofibras de vidrio que, a partir del estudio realizado sobre la exploración del comportamiento de formación de surcos y fractura de la mezcla asfáltica reforzado con esta fibra, se demostró que se mejoró la resistencia a la fractura, como también mejoró la formación de surcos (Morea y Zerbino, 2018). Estas mejoras también se representan bajo la adición de otro tipo de fibra sintética, tal es el caso del uso de fibras plásticas de desecho que, al ser evaluadas a la resistencia de rotura y surco, manifestaron un incremento del desempeño al mejorar estas propiedades y también un impacto positivo al reutilizar los desechos plásticos (Abu Abdo y Jung, 2020).

Por otro lado, está el uso de diversas fibras naturales. Dentro de este tipo se usó las fibras de bambú, que, a partir de este estudio se evaluó la mejora del desempeño con el diseño de dos tipos de mezcla, una patrón y una con adición de fibra, siendo sometidas a pruebas de seguimiento de ruedas y vigas de flexión de tres puntos, se demostró que se fortaleció la conducta de formación de surcos y de agrietamiento a baja temperatura (Sheng et al., 2019). Dentro de la 
misma categoría se hace presente el uso de fibras de yute que, a partir del estudio que indagó la conducta a baja temperatura del cemento asfáltico Warmmix (mezcla producida a $20-25^{\circ} \mathrm{C}$ menos que el cemento asfáltico típico) reforzado con estas fibras, se obtuvo resultados favorables respecto a la mejora de la resistencia a la tracción (Mansourian, Razmi, y Razavi, 2016). En tal sentido, los hallazgos demuestran que se han obtenido mejoras en el desempeño de las mezclas asfálticas al usar fibras naturales y sintéticas como materiales de refuerzo. Adicionalmente, se ha contribuido de manera positiva al medio ambiente al reutilizar materiales de desecho ya sean naturales o sintéticos. Debido a esto se analizó distintos tipos de artículos con el afán de lograr una noción de la optimización de las propiedades de las mezclas asfálticas con adición de fibra. La presente revisión literaria está estructurada bajo los aspectos referentes al tipo de fibras adicionados, los métodos utilizados para evaluar las mezclas con fibras, los porcentajes óptimos de fibras adicionados, las longitudes de las fibras usadas, las propiedades mejoradas en las mezclas con fibras, el desempeño de las mezclas según el tipo de cemento asfaltico, el desempeño de las mezclas según el tipo de mezcla y, finalmente, la rentabilidad de las mezclas fibrosas.

\section{Metodología}

La revisión se realizó utilizando 81 artículos indexados, encontrados en la base de datos Scopus y Ebsco. Se excluyeron todos los artículos con antigüedad mayor a los 7 años. Para la búsqueda se utilizaron las siguientes palabras clave: flexible pavement fiber, pavement with fiber of glass, asphalt mix with fiber, asphalt pavement with fibers, performance of the use of fibers in asphalt mixtures, mix asphalt with fibers, cold mix asphalt using fibers, asphalt mix with natural fibers, asphalt with natural fiber, bitumen with fibers, asphalt bitumen with fibers, asphalt with fibers; incluyendo las áreas temáticas de engineering y materials science, asphalt modifiers, fibers, bituminous materials, cost effectiveness, cold asphalt, natural fibers. La búsqueda de información se realizó desde el 12 de abril hasta el 14 de junio del 2021. Además, se incluyó un resumen de las principales propiedades mejoradas con la adición de fibras en los pavimentos flexibles.

En el Cuadro 1 se muestra con mejor detalle la distribución de las revistas según su base de datos y los años de publicación comprendidos entre el 2014-2021.

En el Cuadro 2 se muestra la búsqueda realizada con minuciosidad, especificando la base de datos consultada, el intervalo de los años de búsqueda, las palabras clave acompañado de su cantidad de documentos encontrados, las áreas temáticas filtradas, de igual forma, la cantidad de documentos encontrados $y$, finalmente, el número de artículos elegidos.

En el Cuadro 3 se muestra un resumen de autores, tipos de fibras, porcentajes óptimos y las principales propiedades mejoradas en los pavimentos flexibles con adición de fibras.

\section{Cuadro 1. Artículos distribuidos según la base de datos y año de publicación}

\begin{tabular}{|c|c|c|c|c|c|c|c|c|c|}
\hline \multirow{2}{*}{$\begin{array}{c}\text { Base de } \\
\text { datos }\end{array}$} & \multicolumn{8}{|c|}{ Año de Publicación } & \multirow{2}{*}{ Total } \\
\hline & 2014 & 2015 & 2016 & 2017 & 2018 & 2019 & 2020 & 2021 & \\
\hline Scopus & 4 & 3 & 4 & 4 & 8 & 11 & 27 & 2 & 63 \\
\hline Ebsco & 2 & 0 & 2 & 1 & 3 & 4 & 6 & 0 & 18 \\
\hline Total & 6 & 3 & 6 & 5 & 11 & 15 & 33 & 2 & 81 \\
\hline
\end{tabular}


Cuadro 2. Criterios de búsqueda para la selección de los artículos usados en la presente investigación

\begin{tabular}{|c|c|c|c|c|c|c|}
\hline $\begin{array}{l}\text { Base de } \\
\text { datos }\end{array}$ & $\begin{array}{l}\text { Años de } \\
\text { búsqueda }\end{array}$ & Ecuación de búsqueda & $\begin{array}{l}\text { Numero de } \\
\text { documentos }\end{array}$ & $\begin{array}{l}\text { Filtro (áreas de } \\
\text { búsqueda) }\end{array}$ & $\begin{array}{c}\text { Resultados } \\
\text { del filtro }\end{array}$ & $\begin{array}{l}\text { Artículos } \\
\text { elegidos }\end{array}$ \\
\hline \multirow{19}{*}{ Scopus } & \multirow{19}{*}{ 2014-2021 } & flexible pavement fiber & 137 & Engineering & 116 & 13 \\
\hline & & \multirow{2}{*}{$\begin{array}{l}\text { pavement with } \\
\text { fiber of glass }\end{array}$} & \multirow[b]{2}{*}{172} & Engineering & \multirow[b]{2}{*}{161} & \multirow[b]{2}{*}{6} \\
\hline & & & & $\begin{array}{l}\text { Materials } \\
\text { Science }\end{array}$ & & \\
\hline & & \multirow[b]{2}{*}{ asphalt mix with fiber } & \multirow[b]{2}{*}{338} & Engineering & \multirow[b]{2}{*}{310} & \multirow[b]{2}{*}{7} \\
\hline & & & & $\begin{array}{l}\text { Materials } \\
\text { Science }\end{array}$ & & \\
\hline & & $\begin{array}{c}\text { flexible } \\
\text { pavement fiber }\end{array}$ & 137 & Engineering & 116 & 1 \\
\hline & & $\begin{array}{c}\text { asphalt pavement } \\
\text { with fibers }\end{array}$ & 679 & Engineering & 565 & 10 \\
\hline & & \multirow[b]{2}{*}{$\begin{array}{l}\text { fiber performance } \\
\text { in asphalt }\end{array}$} & \multirow[b]{2}{*}{684} & Engineering & \multirow[b]{2}{*}{628} & \multirow[b]{2}{*}{6} \\
\hline & & & & $\begin{array}{l}\text { Materials } \\
\text { Science }\end{array}$ & & \\
\hline & & \multirow[b]{2}{*}{ mix asphalt with fibers } & \multirow[b]{2}{*}{338} & Engineering & \multirow[b]{2}{*}{310} & \multirow[b]{2}{*}{7} \\
\hline & & & & $\begin{array}{l}\text { Materials } \\
\text { Science }\end{array}$ & & \\
\hline & & \multirow{2}{*}{$\begin{array}{l}\text { cold mix asphalt } \\
\text { using fibers }\end{array}$} & \multirow[b]{2}{*}{36} & Engineering & \multirow[b]{2}{*}{34} & \multirow[b]{2}{*}{1} \\
\hline & & & & $\begin{array}{l}\text { Materials } \\
\text { Science }\end{array}$ & & \\
\hline & & \multirow{2}{*}{$\begin{array}{l}\text { asphalt mix with } \\
\text { natural fibers }\end{array}$} & \multirow[b]{2}{*}{50} & Engineering & \multirow[b]{2}{*}{44} & \multirow[b]{2}{*}{3} \\
\hline & & & & $\begin{array}{l}\text { Materials } \\
\text { Science }\end{array}$ & & \\
\hline & & \multirow[b]{2}{*}{ asphalt with natural fiber } & \multirow[b]{2}{*}{115} & Engineering & \multirow[b]{2}{*}{101} & \multirow[b]{2}{*}{2} \\
\hline & & & & $\begin{array}{l}\text { Materials } \\
\text { Science }\end{array}$ & & \\
\hline & & bitumen with fibers & 261 & Engineering & 201 & 4 \\
\hline & & asphalt bitumen with fibers & 179 & Engineering & 150 & 3 \\
\hline \multirow{6}{*}{ Ebsco } & \multirow{6}{*}{ 2014-2021 } & & & $\begin{array}{c}\text { asphalt } \\
\text { modifiers }\end{array}$ & 52 & 1 \\
\hline & & & & fibers & 71 & 9 \\
\hline & & asphalt with fibers & 416 & $\begin{array}{c}\text { bituminous } \\
\text { materials }\end{array}$ & 24 & 2 \\
\hline & & & & $\begin{array}{c}\text { cost } \\
\text { effectiveness }\end{array}$ & 3 & 1 \\
\hline & & & & natural fibers & 8 & 3 \\
\hline & & Cold asphalt with fibers & 6 & - & 6 & 2 \\
\hline & & Total & & & & 81 \\
\hline
\end{tabular}




\section{Cuadro 3. Principales propiedades mejoradas con la adición de Fibras en los Pavimentos Flexibles}

\begin{tabular}{|c|c|c|c|c|c|c|c|c|}
\hline \multirow{2}{*}{ Autores/Año } & \multirow{2}{*}{ Fibra } & \multirow{2}{*}{$\begin{array}{l}\text { \% Óptimo } \\
\text { de fibra }\end{array}$} & \multicolumn{6}{|c|}{ Propiedades mejoradas* } \\
\hline & & & A & B & $\mathrm{C}$ & D & E & $F$ \\
\hline (Kamaruddin, Napiah, y Nahi, 2016) & Polipropileno y Poliéster & $0,50 \%$ & sí & NO & NO & - & - & - \\
\hline (Wang, Zhou, Hu, Shen, y Dong, 2021) & Cerámica & $0,40 \%$ & $\mathrm{SI}$ & - & - & - & - & - \\
\hline (Morova et al., 2016) & $\begin{array}{c}\text { Poliparafenilen } \\
\text { tereftalamida polimérica }\end{array}$ & $0,25 \%$ & NO & NO & NO & Sí & - & - \\
\hline (Parimita, 2020) & $\begin{array}{l}\text { Tallo de plátano, } \\
\text { desperdicio de } \\
\text { coco maduro }\end{array}$ & $0,30 \%$ & - & - & sí & sí & - & sí \\
\hline $\begin{array}{l}\text { (Herráiz, Herráiz, Domingo, y Domingo, } \\
\text { 2016) }\end{array}$ & Posidonia & $1,5-2 \%$ & sí & Sí & Sí & - & - & sí \\
\hline (Ziari, Saghafi, Moniri, y Bahri, 2020) & Poliolefina-aramida & $0,05 \%$ & - & Sí & Sí & - & - & Sí \\
\hline (Ziari y Moniri, 2019) & Poliolefina & $0,12 \%$ & - & Sí & - & - & - & Sí \\
\hline (Chen, Yi, Chen, y Feng, 2019) & Tallos de maíz & $9,00 \%$ & Sí & - & - & Sí & - & Sí \\
\hline (Guo, Li, Lu, Bi, y He, 2020) & Basalto, poliéster, lignina & $0,40 \%$ & Sí & - & - & - & Sí & - \\
\hline (Liu, Li, Chen, Guan, y Liu, 2020) & Paja de algodón & $0,20 \%$ & sí & - & - & - & sí & - \\
\hline (Bdour, Khalayleh, y Al-Omari, 2015) & Lana de alambre & $0,50 \%$ & - & - & NO & Sí & - & - \\
\hline (Do, Nguyen, Tran, y Tai Nguyen, 2020) & Forta-fi & $0,10 \%$ & - & Sí & - & sí & - & - \\
\hline (Arabani, Shabani, y Hamedi, 2019) & Cerámica & $3,00 \%$ & - & Sí & Sí & - & - & - \\
\hline (Hao, y Hao, 2014) & Basalto & $0,30 \%$ & Sí & Sí & - & - & Sí & - \\
\hline (Wang, Yang, Zhan, Ding, y Jin, 2018) & Poliacrilonitrilo & $0,30 \%$ & - & Sí & - & - & - & - \\
\hline (Atherton, Ruddock, y Shanbara, 2017) & Coco, vidrio & $0,35 \%$ & - & - & Sí & - & Sí & - \\
\hline (Shanbara, Ruddock, y Atherton, 2018c) & $\begin{array}{l}\text { Vidrio, cáñamo, } \\
\text { yute y bonote }\end{array}$ & $0,35 \%$ & Sí & - & Sí & - & - & - \\
\hline (Takaikaew et al., 2018) & Poliolefina y aramida & $0,05 \%$ & - & Sí & Sí & Sí & - & - \\
\hline (Enieb, Diab, y Yang, 2019) & Vidrio & 0,3 y $0,6 \%$ & Sí & - & Sí & - & - & - \\
\hline (Alnadish, y Aman, 2019) & Aramida & $0,05 \%$ & - & Sí & Sí & - & - & - \\
\hline (Zhang, Hao, Pang, y Mwanza, 2016) & Híbrido & $7,20 \%$ & Sí & - & Sí & - & - & - \\
\hline
\end{tabular}

\section{Resultados y discusión}

El presente artículo reunió información de diferentes estudios comparando resultados y criterios de los diversos autores, con el afán de clasificarlos en 8 apartados, produciendo un intercambio de criterios sobre el uso de distintos tipos de fibras que mejoran las características mecánicas de las mezclas asfálticas.

\section{Tipos de fibras adicionados en las mezclas asfálticas}

\section{Fibras sintéticas}

Kassem y colaboradores afirmaron que son muchas las fibras sintéticas utilizadas en las mezclas asfálticas, dentro de los diferentes tipos utilizados, las fibras de 
aramida y poliolefina tienen un efecto positivo sobre el desempeño de la mezcla asfáltica cuando se usan en las mezclas con un tamaño de agregado máximo nominal grande (Kassem, Saleh, Zalghout, y Chehab, 2018). Además, Bueno y Poulikakos (2020) estudiaron no solo el uso de fibras de aramida y poliolefinas (tipo A), sino también de fibras de poliacrilonitrilo (tipo B), demostrando la idoneidad de su uso en las mezclas asfálticas, al mostrar efectos positivos superiores al de una mezcla ordinaria.

Kamaruddin y colaboradores mencionaron que las fibras sintéticas son utilizadas con el fin de actuar como modificadores de las mezclas asfálticas, presentándose como posibles modificadores usaron dos tipos de fibras poliméricas (fibra de polipropileno y fibra de poliéster), las cuales demostraron un efecto positivo al ser adicionadas en las mezclas, favoreciendo en la reducción del daño por humedad (Kamaruddin et al., 2016). Así mismo, Morova y colaboradores mejoraron la misma característica usando fibra polimérica poliparafenileno tereftalamida bajo un contenido óptimo de 0,25\% (Morova, et al., 2016). Además, Wang y colaboradores mostraron que otro posible modificador sintético a utilizar es la fibra de cerámica, debido a que mejoró sustancialmente las propiedades mecánicas, en comparación con las mezclas ordinarias (Wang, Zhou, Hu, Shen, y Dong, 2021).

Cierto tipo de materiales son característicos debido a su resistencia, pero de manera independiente, el combinarlos con otros componentes resulta muy tentativo, en tal sentido Çetin (2014) investigó el uso fibras de acero en las mezclas asfálticas, demostrando un desempeño adecuado tanto en el cementoasfáltico como en la capa de desgaste bajo proporciones de $0,1 \%$ y $5 \%$ de fibra. Complementariamente, en su investigación Jasni y colaboradores mostraron que la inclusión de fibra de acero en la mezcla aumenta la estabilidad, el módulo elástico y la fluencia dinámica mostrando mejores resultados bajo el contenido óptimo de fibra de $0,4 \%$ (Jasni et al., 2020).

\section{Fibras naturales}

Parimita (2020) investigó el uso de las fibras recolectadas del tallo de plátano y de la parte de desecho del coco maduro, mostrando que su uso proporciona mejor capacidad de resistir la formación de surcos y el agrietamiento. De manera complementaria, en su investigación Colares do
Vale y colaboradores investigaron la incorporación de fibras de coco en mezclas asfálticas discontinuas, generando características satisfactorias en la susceptibilidad a la humedad y la resistencia a la tracción (Colares do Vale, Toé Casagrande, y Barbosa Soares, 2014).

Por otra parte, Aljubory y colaboradores investigaron el uso de fibras de palma en mezclas asfálticas, las cuales mejoran la resistencia a la tracción indirecta al aumentar el contenido de fibra de $0 \%$ a $1 \%$ (Aljubory, Abbas, y Bdan, 2020). Propiedad que Kar y colaboradores también investigaron, y llegaron a concluir que es mejorada mediante la adición de $0,3 \%$ de fibra de sisal, pero en una mezcla con cenizas volantes como relleno (Kar, Giri, y Panda, 2019). Pirmohammad y colaboradores demostraron que también está presente la mejora de la resistencia a la fractura, estudiando la adición de fibras de kenaf y lana de cabra, mostraron que se mejora la resistencia a la fractura dependiendo de la longitud y el contenido de fibra utilizada (Pirmohammad, Majd Shokorlou, y Amani, 2020). Adicionalmente, Kara De Maeijer y colaboradores en su investigación mostraron que otra característica a optimizar con el uso de fibras naturales es el drenaje del cemento asfáltico, el cual se reduce al añadir fibra de turba natural de manera controlada con el fin de evitar dificultades en la compactación de estas mezclas (Kara De Maeijer et al., 2019).

Muy alejado de las fibras naturales usadas comúnmente, Herráiz y colaboradores estudiaron el uso de fibra de posidonia oceánica en el cemento asfáltico, presentando características adecuadas cuando el contenido de fibra se encuentra entre 1,5\% y $2 \%$, mejorando la rigidez, la vida útil a la fatiga y la formación de surcos (Herráiz, Herráiz, Domingo, y Domingo, 2016).

\section{Métodos utilizados para evaluar las mezclas asfálticas con fibras}

Son diversos los métodos que se utilizan para evaluar las mezclas asfálticas con fibras, una de ellas es la prueba Marshall, la cual es simple, económica y se distingue por tener dos características principales, el análisis de vacíos con aire y resultados de estabilidad y flujo Marshall (Mrema, Noh, Kwon, y Lee, 2020). En relación con la estabilidad de flujo, Preciado y 
colaboradores mencionan que ésta se expresa como el cociente de Marshall, el cual es un indicador de la rigidez de la mezcla. Es decir, que los altos valores del cociente de Marshall indican una mezcla de alta rigidez con mayor capacidad para distribuir la carga aplicada (Preciado et al., 2017).

Otra prueba muy utilizada para evaluar el desempeño de las mezclas asfálticas es la prueba de tracción indirecta, la cual se utiliza de manera típica para encontrar la resistencia a la tracción de la muestra de la mezcla compactada (Al-Bdairi, Al-Taweel, y Noor, 2020), dentro de las propiedades de tracción que se evalúan se incluyen la resistencia a la tracción, tenacidad y deformación (desplazamiento) (Mrema, Noh, Kwon, y Lee, 2020).

La prueba de susceptibilidad a la humedad de las mezclas, definida como la alteración de la mezcla asfáltica por el agua, es decir que, si el agua penetra en la mezcla asfáltica, daña la unión entre los agregados y el cemento asfáltico, acelerando así el deterioro del pavimento (Çetin, Evirgen, Karslioglu, y Tuncan, 2020). En esta prueba, Park y colaboradores mencionaron que la resistencia a la humedad de la mezcla asfáltica se determina comparando la resistencia a la tracción indirecta en estado seco y después del estado de congelación-descongelación de agua (Park, Shoukat, Yoo, y Lee, 2020).

La prueba de ductilidad indica el desempeño del cemento asfáltico a alta y baja temperatura. Esta prueba se realiza para medir la longitud de estiramiento de las muestras del cemento asfáltico base y modificado (Sabaeei, Napiah, Sutanto, y Alaloul, 2019).

La prueba de resistencia a la formación de surcos, consta de realizar una prueba de seguimiento de rueda con el objeto de examinar la estabilidad dinámica de la mezcla asfáltica, que viene a ser el índice de resistencia a la formación de surcos causado por los efectos de la fuerza de corte impartida por las ruedas de los vehículos a alta temperatura (Kim, Kim, Yoo, y Shin, 2018). Preciado y colaboradores mencionaron que otra prueba que busca el mismo fin es la prueba de carga repetida; la cual se utiliza para determinar la deformación permanente de las mezclas asfálticas, que se relaciona con el potencial de formación de surcos (Preciado et al., 2017).
Por otro lado, con el fin de determinar la longitud de fibra favorable, se realiza la prueba de penetración del cono, en conjunto con la prueba de estirado de la canasta de malla. La primera evalúa la resistencia al flujo y al cizallamiento del cemento asfáltico reforzado con fibra, y la otra determina la adsorción de fibras en el cemento asfáltico y la estabilización del cemento asfáltico reforzado con fibra (Guo, Li, $\mathrm{Lu}, \mathrm{Bi}, \mathrm{y} \mathrm{He}, 2020)$. Mientras que la prueba de barrido de secuencia usando un reómetro de cizallamiento dinámico se usa para investigar los efectos de los métodos de tratamiento de fibras en las propiedades reológicas de las emulsiones asfálticas reforzadas con fibras (Liu et al., 2020).

\section{Porcentajes óptimos de fibras en las mezclas asfálticas}

Ziari y colaboradores utilizaron las fibras de poliolefina-aramida como aditivos en porcentajes de $0,025 \%, 0,05 \%$ y $0,075 \%$ en relación al peso de la mezcla, recomendándose el uso de $0,075 \%$ de fibras cuando se presenten problemas de surcos o grietas y el 0,05\% de fibras para la mejora de la resistencia a la fatiga (Ziari, Saghafi, Moniri, y Bahri, 2020). Mientras que Alnadish y Aman (2019) analizaron solo las fibras de aramida en proporciones de 0,$05 ; 0,1 ; 0,2$ y $0,3 \%$ en relación al peso total de agregado, sobresaliendo el primer porcentaje evaluado de 0,05\%, debido a que aumentó la vida útil bajo esta proporción.

Siguiendo con el uso de fibras sintéticas, Ziari y Moniri (2019) investigaron el uso de fibras de vidrio de poliolefina en las mezclas asfálticas, utilizando cantidades de $0,06 \% ; 0,12 \%$ y $0,18 \%$ en peso de la mezcla total, obteniendo mejores resultados bajo la proporción de $0,12 \%$ de fibra. Entretanto, Eisa y colaboradores evaluaron el efecto de la fibra de vidrio sobre las características de una mezcla asfáltica en caliente, mostrando que la mezcla con $0,25 \%$ de fibras por peso total de la mezcla exhibió valores positivos para casi todas las propiedades evaluadas (Eisa, Basiouny, y Daloob, 2021).

Por otro lado, Chen y colaboradores investigaron dentro del uso de fibras naturales el uso de fibras de tallos de maíz, las cuales se usaron en porcentajes de $0 \%, 2 \%, 4 \%, 6 \%, 8 \%$ y $10 \%$ en relación al peso del cemento asfáltico, influyendo de manera positiva al comportamiento a baja temperatura del cemento 
asfáltico bajo la adición de $2 \%$ de fibras (Chen, Yi, Chen, y Feng, 2019). Asimismo, Li y colaboradores investigaron a las fibras de bagazo de caña las cuales, usadas en un porcentaje de $0,2 \%$ en relación con el peso total de la mezcla, mostraron que se mejoró enormemente la estabilidad a alta temperatura y la resistencia al agrietamiento a baja temperatura de las mezclas asfálticas (Li et al., 2020).

\section{Longitudes óptimas de las fibras usadas en las mezclas asfálticas}

Basándose en el uso de fibras sintéticas, Kassem y colaboradores estudiaron el uso de fibras de aramida y poliolefina con dimensiones de $19 \mathrm{~mm}$ de largo, logrando obtener un efecto positivo sobre el desempeño de la mezcla asfáltica (Kassem, Saleh, Zalghout, y Chehab, 2018). Bajo el mismo concepto, Mawat y Ismael (2020) en su investigación presentaron el uso de las fibras de carbono con longitud de $2 \mathrm{~cm}$ y un $0,30 \%$ en relación al peso de la mezcla, mostrando resultados positivos en relación a la capacidad de resistir a la tracción indirecta y al índice de resistencia retenida. Como complemento a esta, la investigación de Aboutalebi y Namavar (2020), que utilizó fibras de parafina con longitud optima de $12 \mathrm{~mm}$ bajo un contenido de fibra de 1\% (valor óptimo del estudio) mejoró con creces las propiedades mecánicas de las mezclas asfálticas.

Desde la perspectiva del uso de fibras naturales, Lou y colaboradores utilizaron fibras de basalto en longitudes de $3 ; 6 ; 9 ; 12$ y $15 \mathrm{~mm}$ sobre mezclas asfálticas, encontrando que la adición de fibras mejora en grandes proporciones el desempeño de las mezclas bajo las longitudes de 6; 9 y 12 mm (Lou, Xiao, Kang, Wu, y Lu, 2020). Compartiendo la categoría de fibras naturales, Maharaj y colaboradores emplearon fibras de coco añadidas de manera gradual de $1 \%$ a $8 \%$ en peso del cemento asfáltico, con longitudes de 2,$5 ; 5 ; 7,5$ y 10 mm, logrando alcanzar una rigidez significativa y mayor elasticidad bajo porcentajes de fibra de $6 \%$ y $8 \%$ con una longitud de 2,5mm (Maharaj, Ali, Ramlochan, y Mohamed, 2019). Asimismo, Ramalingam y colaboradores estudiaron la aplicación de fibras de sisal en mezclas bituminosas, demostrando la mejora de la vida útil bajo la adición de $0,05 \%$ de fibra (en peso del agregado) con una longitud de $15 \mathrm{~mm}$ (Ramalingam, Murugasan, y
Nagabhushana, 2017). De igual importancia, en su estudio Ferreira da Costa y colaboradores mostraron que el uso de fibras del pseudotallo de la planta de banano con un tamaño de fibra de $20 \mathrm{~mm}$ en mezclas asfálticas, demuestran características superiores en comparación con las mezclas sin fibras (Ferreira da Costa, Grangeiro de Barros, Lucena Lopes, y Lucena Lopes, 2020).

\section{Uso de fibras para la mejora de las propiedades mecánicas de las mezclas asfálticas}

Javani y colaboradores investigaron dentro del uso de fibras sintéticas el uso de fibras de polipropileno y vidrio reciclado para mejorar las características de las mezclas asfálticas, exhibiendo buena capacidad de tolerar la formación de surcos, vida prolongada a la fatiga y menos agrietamiento por reflexión (Javani, Kashi, y Mohamadi, 2019). Siguiendo con uso de fibras sintéticas, Wiśniewski y colaboradores en su estudio mostraron que las fibras de aramida en la mezcla asfáltica de alto módulo reducen ligeramente el contenido de vacíos de aire (Wiśniewski, Słowik, Kempa, Lewandowska, y Malinowska, 2020). De manera adicional, Dehghan y Modarres (2017) investigaron el uso de fibras de tereftalato de polietileno, las cuales bajo porcentajes de adición de $1 \%$ y $2 \%$ demostraron una vida útil mayor al de la mezcla patrón.

La mejora de las propiedades mecánicas también se presentó con el uso de fibras naturales, ejemplo de ello es el estudio de Pirmohammad y Hojjati, (2020) mostró que el uso de fibras de kenaf y basalto como refuerzo de las mezclas asfálticas en caliente, mejoraron la resistencia a la factura de las mezclas asfálticas. Adicionalmente, Liu y colaboradores indagaron sobre el uso de las fibras de paja de algodón, las cuales incrementaron la estabilidad bajo condiciones de temperaturas altas ( $\mathrm{Liu}, \mathrm{Li}, \mathrm{Chen}$, Guan, y Liu, 2020).

Por otra parte, Bdour y colaboradores estudiaron el uso de una fibra inusual llamada fibra de lana de alambre, la cual incrementó la cualidad de soportar la tracción de las mezclas asfálticas, pero aumentó la formación de surcos, siendo recomendable para áreas donde la formación de surcos no es una falla predominante (Bdour, Khalayleh, y Al-Omari, 2015). 
Adicionalmente, Do y colaboradores estudiaron el uso de fibras Forta-fi, la cuáles bajo un porcentaje de $0,1 \%$ de fibra en relación al peso total de la mezcla, aumentaron la vida útil a la fatiga del cemento asfáltico (Do, Nguyen, Tran, y Tai, 2020). Adicionalmente, Desseaux y colaboradores en su estudio utilizaron fibras de celulosas aceitada, las cuales modificaron las propiedades mecánicas del cemento asfáltico de una manera distinta a los aditivos comunes (Desseaux et al., 2018).

\section{Desempeño de las mezclas asfálticas con fibras según el tipo de cemento asfáltico}

Naseri y colaboradores presentaron en su estudio el uso de la fibra de cerámica (CF) como modificador del cemento asfáltico (PG 64-22), demostrando que al usarlas hasta un $0,4 \%$ en peso del cemento asfáltico se redujo la rigidez a la fluencia e incremento el beneficio-costo, haciendo posible que las características reológicas del cemento asfáltico sean adecuadas para un grado más bajo que el cemento asfáltico de control (Naseri Yalghouzaghaj, Sarkar, Hamedi, y Hayati, 2020). Arabani y colaboradores estudiaron el mismo tipo de fibra, pero con un cemento asfáltico PG 64-16 (más utilizados para climas cálidos y moderados) obteniendo que la vida útil a la fatiga de la mezcla asfáltica aumentó al agregarle hasta un 3\% de CF, pero si le agrega CF por encima del $3 \%$ ésta disminuyó la vida útil a la fatiga (Arabani, Shabani, y Hamedi, 2019).

Por otro lado, también está el uso de fibras naturales, en donde Hao y Hao (2014) demostraron que el uso de fibras de basalto en cemento asfáltico SK 70 bajo el mismo nivel de estrés duplican la vida de fatiga de la mezcla asfáltica.

En otra instancia, Wang y colaboradores indagaron el uso fibras sintéticas en un cemento asfáltico con grado de penetración 70/100, adicionando fibra de poliacrilonitrilo, mostrándose mejoras en la propiedad de antifatiga, (Wang, Yang, Zhan, Ding, y Jin, 2018). Bajo el uso el mismo betún, Geckil y Ahmedzade (2020) estudiaron la mezcla asfáltica con adición de fibra de carbono, obteniendo que al adicionar fibra de carbono aumenta la resistencia al esfuerzo cortante en un $25 \%$, la vida útil a la fatiga en un $51 \%$ y la resistencia a la deformación permanente en 2,25 veces. Continuando con las fibras sintéticas, García y colaboradores investigaron la adición de fibras de lana de acero al mismo cemento asfáltico dando como resultado que esta no mejora las propiedades mecánicas de la mezcla asfáltica, incluso estas pueden llegar a cambiar la distribución de vacíos de aire de la mezcla lo que podría ocasionar que la capacidad de resistir la pérdida de partículas se vea inmiscuida (García, Norambuena Contreras, y Partl, 2014).

Dentro de las fibras no muy usuales Rahman y colaboradores utilizaron fibras de colillas de cigarrillos (CB), incorporándolo a distintos tipos de cemento asfáltico tales como C320, C170 y PMB $A 10 E$, obteniendo que el cemento asfáltico C320 y PMB A10E son los más apropiados para ser modificados con CB sin comprometer la propiedad de penetración (Rahman, Mohajerani, y Giustozzi, 2020).

\section{Desempeño de las mezclas asfálticas con fibras según el tipo de mezcla asfáltica}

\section{Mezcla asfáltica en frío}

Ferrotti y colaboradores investigaron una mezcla asfáltica en frío con el uso de fibras (celulosa, vidrio-celulosa, nylon-poliéster-celulosa), siendo dosificados en $0,15 \%$ y $0,30 \%$ por el peso del agregado, encontrando que las fibras de celulosa al $0,15 \%$ mostraron mejores resultados respecto a la mezcla sin fibras, destacando un mayor desempeño en tiempos de curado más cortos (Ferrotti, Pasquini, y Canestrari, 2014). Asimismo, Atherton y colaboradores indagaron sobre el uso de fibras de bonote y vidrio, que al ser utilizados como materiales de refuerzo, optimizaron la mezcla asfáltica en frío (CMA) al mejorar de manera relevante el módulo de rigidez y la resistencia a la tracción de la mezcla (Atherton, Ruddock, y Shanbara, 2017). Pero al centrarse solo en la utilización de fibras naturales Shanbara y colaboradores utilizaron fibras de bonote y yute como reforzamiento en mezclas CMA, observándose que estas muestran efectividad para disminuir la formación de surcos, catalogándolas como componentes de refuerzo que pueden aumentar la vida útil del pavimento (Shanbara, Ruddock, y Atherton, 2018a). 
Por otra parte, con el fin de caracterizar el comportamiento de formación de surcos en CMA, Shanbara y colaboradores usaron fibras de vidrio y de cáñamo, resultando que el uso de estas fibras mejoró el desempeño de las mezclas CMA frente a la deformación permanente (Shanbara, Ruddock, y Atherton, 2018b). En busca de reforzar la investigación anterior, Shanbara y colaboradores utilizaron fibras de vidrio, cáñamo, yute y bonote en CMA, mediante el cual determinaron que las mezclas CMA mejoradas con fibras naturales y sintéticas facilitaron una deformación permanente sustancialmente menor que las mezclas tradicionales frías y calientes (Shanbara, Ruddock, y Atherton, 2018c). En relación con el uso del mismo tipo de fibras Shubbar y colaboradores estudiaron las mezclas CMA, denotando que las mezclas asfálticas en frío pueden ser mejoradas de manera general al incorporar fibras naturales y sintéticas (Shubbar, Shanbara, Ruddock, y Atherton, 2020).

Diversificando el estudio de adición de fibras en una mezcla CMA, Martinez y colaboradores usaron fibras F1 (malla de polipropileno) y F2 (polipropileno monofilamento) con los betunes, Betún A (Bit $A$ ), Betún $B$ (Bit B) y Betún C (Bit $C$ ), resultando que los contenidos óptimos de fibra fueron $0,075 \%$ (en masa de agregados) para F1 y 0,15\% para F2, demostrando que estas fibras incrementaron las propiedades mecánicas a temperaturas bajas e intermedias (Martinez, Giustozzi, Crispino, y Flintsch, 2015).

\section{Mezcla asfáltica en caliente}

Alfalah y colaboradores en su estudio usaron fibras de vidrio, basalto, carbono y aramida en proporciones de $0,16 \%$ (fibra de vidrio, basalto y carbono) y $0,05 \%$ (aramida) por peso total de la mezcla, logrando alcanzar la mejora del desempeño en formación de surcos y durabilidad de las mezclas asfálticas en caliente (HMA) (Alfalah et al., 2020). Por otro lado, Miao y colaboradores estudiaron el uso de cuatro tipos de fibra, incluido un plástico reforzado con fibra (FRP), dos fibras de lignina y una fibra de basalto, destacando que las fibras de basalto presentaron el mejor efecto de refuerzo en HMA al contar con una alta energía superficial (Miao, Wang, y Wang, 2019). Además del uso de estas fibras, Takaikaew y colaboradores utilizaron las fibras de aramida y poliolefina como material de refuerzo en HMA, las cuales exhibieron un desempeño por encima de la mezcla asfáltica ordinaria, presentando así una vida útil más larga (Takaikaew et al., 2018).

Centrándose solo en las fibras sintéticas, Meng y colaboradores indagaron sobre el uso de fibras de poliéster en mezclas asfálticas en caliente, mostrando un desempeño de fatiga relativamente bueno con un contenido de fibra de $0,35 \%$ y una relación de longitud a diámetro de 324 (Meng, Gao, Chen, y Huang, 2020). Además, Enieb, Diab, y Yang estudiaron el uso de fibras de vidrio en mezclas HMA las cuales pudieron impartir actitudes positivas como aumentar la fuerza, resistir a la creación de surcos, menor susceptibilidad a ser dañado por humedad, retardar el agrietamiento por fatiga, mejorar la capacidad de curación y combatir los cambios adversos del envejecimiento (Enieb, Diab, y Yang, 2019). Mientras que Koçkal y Köfteci (2016) intentaron minimizar el efecto ambiental agresivo sobre la mezcla asfáltica en caliente, utilizando fibras de polipropileno en un $7,5 \%$, obteniendo con esto, un valor óptimo de la estabilidad Marshall.

\section{Rentabilidad del uso de fibras en las mezclas asfálticas}

Alnadish y Aman (2019) mediante su estudio determinaron que el uso de fibras de polipropileno en HMA incrementaron considerablemente el costo inicial, pero este a la vez aumentó el ciclo de vida del pavimento, lo cual a largo plazo sería más económico (Adnan, Uneb, y Sajjad, 2016). También está presente el estudio de Souliman y colaboradores, que investigó el uso de fibras de aramida que, al adicionarlas en un $0,05 \%$ en relación al peso total del agregado, aumentó la resistencia a agrietamiento por fatiga y a la formación de surcos, alargando su vida útil, y a la vez minimizando costos de rehabilitación y mantenimiento. En tal sentido, adicionalmente se demostró que la rentabilidad de la mezcla reforzada con fibras fue 4,25 veces mayor que la de la mezcla controlada sin modificar según el análisis de fatiga, mientras que la rentabilidad de la mezcla reforzada con fibras fue 4,52 veces mayor que la de la mezcla controlada sin modificar según el análisis de formación de surcos (Souliman, Tripathi, y Isied, 2019).

Zhang y colaboradores, estudiaron el uso de fibras combinadas, esta fibra híbrida está compuesta por fibras de lignina, poliéster y polipropileno, y 
demostraron que su beneficio-costo es mejor que el de cada fibra por separado, mostrándose como una opción para reforzar las mezclas asfálticas (Zhang, Hao, Pang, y Mwanza, 2016). Complementariamente, Perca y colaboradores indagaron sobre el uso de fibras de polímero, que mediante un análisis de beneficiocosto se demostró que las fibras de polímero podrían ser una alternativa valiosa, debido a su menor costo en comparación con las mezclas tradicionales (Perca, Hashemian, y Sha, 2020)

\section{Conclusiones}

Mediante la revisión literaria de las investigaciones encontradas de los diferentes autores sobre la mejora de las propiedades mecánicas de las mezclas asfálticas con adición de fibras se han llegado a las siguientes conclusiones:

Se concluyó que dentro que los diferentes tipos de fibras utilizadas en las mezclas asfálticas, se presentó el uso de distintos tipos de fibras sintéticas y fibras naturales con afán de la mejora de las distintas características de las mezclas, resaltando por parte de las fibras sintéticas, las fibras de aramida, mientras que las fibras naturales, las fibras de basalto.

Las fibras son una buena opción de refuerzo a los pavimentos flexibles, ya que en las pruebas realizadas tales como: Marshall, tracción indirecta, penetración del cono, prueba de seguimiento de ruedas, se obtuvieron buenos resultados.

En cuanto al porcentaje óptimo de fibras que se necesitó para mejorar las propiedades de las mezclas asfálticas, estas varían según el tipo de fibra, forma, tamaño y como se añaden en las mezclas, ya que dependiendo de la investigación se puede añadir reemplazando en porcentaje o peso, del agregado fino, agregado grueso, del cemento asfáltico, componentes de la mezcla asfáltica.

El adicionar diferentes tipos de fibras a las mezclas asfálticas, se mejoró distintas propiedades tales como: susceptibilidad a la humedad, resistencia a la fatiga, resistencia a la deformación, resistencia a la tracción, estabilidad a alta temperatura, resistencia al agrietamiento. Siendo la resistencia a la formación de surcos la propiedad que más destacó en las diferentes investigaciones. Pero no todas las fibras utilizadas llegaron a cumplir el objetivo de modificar de manera positiva las propiedades del pavimento, algunas llegaron a afectar la unión entre los agregados y el cemento asfáltico, disminuyendo el tiempo de vida útil del pavimento.

La adición de fibras en mezclas asfálticas en caliente, muestran características que superan con creces a las mezclas asfálticas ordinarias, mientras que el uso de fibras en mezclas asfálticas en frío solo llega a mostrar características que igualan o superan por poco a las mezclas ordinarias.

Al evaluar el uso de fibras en las mezclas asfálticas mediante un análisis de beneficio-costo, se demostró que el uso de fibras resulta una valiosa alternativa en comparación con las mezclas tradicionales. Si bien es cierto muestran un aumento en el costo inicial, pero también un incremento en el ciclo de vida, siendo así económicamente factibles para proyectos viales que se realicen con el de uso pavimentos flexibles.

Con base en la revisión bibliográfica realizada, se recomienda proseguir con estudios encaminados a utilizar fibras en la fabricación de mezclas asfálticas debido a las mejoras que proporciona.

\section{Referencias}

Aboutalebi, E. M., y Namavar , J. M. (2020). Optimum parafibre length according to mechanical properties in hot mix asphalt. Road Materials and Pavement Design, 21(3), 683-700. DOI: 10.1080/14680629.2018.1527240

Abu Abdo, A., y Jung, S. (2020). Investigation of reinforcing flexible pavements with waste plastic fibers in Ras Al Khaimah, UAE. Road Materials and Pavement Design, 21(6), 17531762. DOI: $10.1080 / 14680629.2019 .1566086$

Adnan, Q., Uneb, G., y Sajjad, A. (2016). Comparison of SBS and PP fibre asphalt modifications for rutting potential and life cycle costs of flexible pavements. Road Materials and Pavement Design, 19(2), 484-493. DOI: 10.1080/14680629.2016.1259124

Al-Bdairi, A. S., Al-Taweel, H. M., y Noor, H. M. (2020). Improving the properties of asphalt mixture using fiber materials. Materials Science and Engineering Conference Series, 870, 012092. DOI: 10.1088/1757-899X/870/1/012092 
Alfalah, A., Offenbacker, D., Ali, A., DeCarlo, C., Lein, W., Mehta, Y., y Elshaer, M. H. (2020). Assessment of the Impact of Fiber Types on the Performance of Fiber-Reinforced Hot Mix Asphalt. Transportation Research Record, 2674(4), 337347. DOI: $10.1177 / 0361198120912425$

Aljubory, A., Abbas, A. S., y Bdan, A. (2020). Effect of Palm Fibers on Asphalt Pavement Properties. Materials Science and Engineering Conference Series, 881, 012176. DOI:10.1088/1757$899 X / 881 / 1 / 012176$

Alnadish, A. M., y Aman, M. Y. (2019). Evaluation of aramid fibre-reinforced asphalt mixtures. Lecture Notes in Civil Engineering, 9, 1377-1388. DOI: 10.1007/978-981-10-8016-6_99

Al-Sabaeei, A., Napiah, M. B., Sutanto, M. H., Alaloul, W. S., Zoorob, S. E., y Usman, A. (2020). Influence of nanosilica particles on the high-temperature performance of waste denim fibre-modified bitumen. International Journal of Pavement Engineering. DOI: 10.1080/10298436.2020.1737060

Arabani, M., Shabani, A., y Hamedi, G. H. (2019). Experimental Investigation of Effect of Ceramic Fibers on Mechanical Properties of Asphalt Mixtures. Journal of Materials in Civil Engineering, 31(9). DOI: 10.1061/(ASCE) MT.1943-5533.0002821

Atherton, W., Ruddock, F. M., y Shanbara , H. K. (2017). Improving the Mechanical Properties of Cold Mix Asphalt Mixtures Reinforced by Natural and Synthetic Fibers. International Conference on Highway Pavements and Airfield Technology 2017, 102-111. DOI: $10.1061 / 9780784480946.010$

Bdour, A., Khalayleh, Y., y Al-Omari, A. A. (2015). Assessing mechanical properties of hot mix asphalt with wire wool fibers. Advances in Civil Engineering, 2015, 795903. DOI:10.1155/2015/795903

Bueno, M., y Poulikakos, L. (2020). Chemo-Mechanical Evaluation of Asphalt Mixtures Reinforced With Synthetic Fibers. Frontiers in Built Environment, 6, 41. DOI: 10.3389/fbuil.2020.00041
Çetin, S. (2014). Evaluation on the usability of structure steel fiber-reinforced bituminous hot mixtures. Construction and Building Materials, 64, 414420. DOI: 10.1016/j.conbuildmat.2014.04.093

Çetin, A., Evirgen, B., Karslioglu, A., y Tuncan, A. (2020). The effect of basalt fiber on the performance of stone mastic asphalt. Periodica Polytechnica Civil Engineering, 65(1), 299-308. DOI: 10.3311/ PPci.14190

Chen, Z., Yi, J., Chen, Z., y Feng, D. (2019). Properties of asphalt binder modified by corn stalk fiber. Construction and Building Materials, 212, 225235. DOI: 10.1016/j.conbuildmat.2019.03.329

Colares do Vale, A., Toé Casagrande, M., y Barbosa Soares, J. (2014). Behavior of Natural Fiber in Stone Matrix Asphalt Mixtures Using Two Design Methods. Journal of Materials in Civil Engineering, 26, 457-465. DOI: 10.1061/(ASCE) MT.1943-5533.0000815

Dehghan, Z., y Modarres, A. (2017). Evaluating the fatigue properties of hot mix asphalt reinforced by recycled PET fibers using 4-point bending test. Construction and Building Materials, 139, 384-393. DOI: 10.1016/j. conbuildmat.2017.02.082

Desseaux, S., dos Santos, S., Geiger, T., Tingaut, P., Zimmermann, T., Partl, M., y Poulikakos, L. (2018). Improved mechanical properties of bitumen modified with acetylated cellulose fibers. Composites Part B: Engineering, 140, 139144. DOI: 10.1016 / j.compositesb.2017.12.010

Do, T. T., Nguyen, D. L., Tran, V.T., y Tai Nguyen, H. (2020). Effects of Forta-fi Fiber on the Resistance to Fatigue of Conventional Asphalt Mixtures. 5th International Conference on Green Technology and Sustainable Development, 312-316. DOI: 10.1109/GTSD50082.2020.9303123

Eisa, M., Basiouny, M., y Daloob, M. (2021). Effect of adding glass fiber on the properties of asphalt mix. International Journal of Pavement Research and Technology., 14(4), 403-409. DOI: 10.1007/ s42947-020-0072-6 
Enieb, M., Diab, A., y Yang, X. (2019). Short- and long-term properties of glass fiber reinforced asphalt mixtures. International Journal of Pavement Engineering, 22(1), 64-67. DOI: $10.1080 / 10298436.2019 .1577421$

Ferreira da Costa, L., Grangeiro de Barros, A., Lucena Lopes, A.D., y Lucena Lopes, L.D. (2020). Asphalt mixture reinforced with banana fibres. Road Materials and Pavement Design, 22(8), 18811893. DOI: $10.1080 / 14680629.2020 .1713866$

Ferrotti, G., Pasquini, E., y Canestrari, F. (2014). Experimental characterization of highperformance fiber-reinforced cold mix asphalt mixtures. Construction and Building Materials, 57, 117-125. DOI: 10.1016/j. conbuildmat.2014.01.089

García, A., Norambuena Contreras, J., y Partl, M. (2014). A parametric study on the influence of steel wool fibers in dense asphalt concrete. Materials and Structures, 47(9), 1559-1571. DOI: 10.1617/s11527-013-0135-0

Geckil, T., y Ahmedzade, P. (2020). Effects of carbon fibre on performance properties of asphalt mixtures. The Baltic Journal, 15(2), 49-65. DOI: 10.7250/bjrbe.2020-15.472

Guan, B., Liu, J., Wu, J., Liu, J., Tian, H., Huang, T., Chengcheng, L., y Ren, T. (2019). Investigation of the performance of the ecofriendly fiberreinforced asphalt mixture as a sustainable pavement material. Advances in Materials Science and Engineering, 2019, 6361032. DOI: $10.1155 / 2019 / 6361032$

Guo, Q., Li, L., Cheng, Y., Jiao, Y., y Xu, C. (2015). Laboratory evaluation on performance of diatomite and glass fiber compound modified asphalt mixture. Materials and Design, 66, 5159. DOI: 10.1016/j.matdes.2014.10.033

Guo, F., Li, R., Lu , S., Bi, Y., y He, H. (2020). Evaluation of the Effect of Fiber Type, Length, and Content on Asphalt Properties and Asphalt Mixture Performance. Materials, 13(7), 1556-1579. DOI: 10.3390/ma13071556
Hao, M., y Hao, P. (2014). Natural mineral fiber improved asphalt mixture performance. Applied Mechanics and Materials, 638-640, 1166-1170. DOI: 10.4028/www.scientific.net/ AMM.638-640.1166

Herráiz, T., Herráiz, J., Domingo, L., y Domingo, F. (2016). Posidonia oceanica used as a new natural fibre to enhance the performance of asphalt mixtures. Construction and Building Materials, 102, 601-612. DOI: 10.1016/j. conbuildmat.2015.10.193

Jasni, N., Masri, K., Ramadhansyah, P., Arshad, A., Shaffie, E., Ahmad, J., y Norhidayah, A. (2020). Mechanical Performance of Stone Mastic Asphalt Incorporating Steel Fiber. Materials Science and Engineering Conference Series, 712(1), 012026. DOI: 10.1088/1757899X/712/1/012026

Javani, M., Kashi, E., y Mohamadi, S. (2019). Effect of polypropylene fibers and recycled glass on AC mixtures mechanical properties. International Journal of Pavement Research and Technology, 12(5), 464-471. DOI: 10.1007/s42947-0190056-6

Kamaruddin, I., Napiah, M., y Nahi, M. H. (2016). The Influence of Moisture on the Performance of Polymer Fibre-Reinforced Asphalt Mixture. MATEC Web of Conferences, 78, 01040. DOI: $10.1051 /$ matecconf/20167801040

Kar, D., Giri, J. P., y Panda, M. (2019). Performance Evaluation of Bituminous Paving Mixes Containing Sisal Fiber as an Additive. Transportation Infrastructure Geotechnology, 6(3), 189-206. DOI: 10.1007/s40515-01900079-6

Kar, S. S., Nagabhushana, M. N., y Jain, P. K. (2019). Performance of hot bituminous mixes admixed with blended synthetic fibers. International Journal of Pavement Research and Technology, 12, 370-379. DOI: 10.1007/s42947-019-0044-x

Kara De Maeijer, P., Soenen, H., Van den Bergh, W., Blom, J., Jacobs, G., y Stoop, J. (2019). Peat fibers and finely ground peat powder for application in asphalt. Infrastructures, 4(1), 3. DOI: 10.3390/infrastructures4010003 
Kassem, H., Saleh, N., Zalghout, A., y Chehab, G. (2018). Advanced characterization of asphalt concrete mixtures reinforced with synthetic fibers. Journal of Materials in Civil Engineering, 30(11), 04018307. DOI: 10.1061/(ASCE) MT.1943-5533.0002521

Kim, M.-J., Kim, S., Yoo, D.-Y., y Shin, H.-O. (2018). Enhancing mechanical properties of asphalt concrete using synthetic fibers. Construction and Building Materials, 178, 233-24. DOI: 10.1016/j.conbuildmat.2018.05.070

Klinsky Gutiérrez, L. M., Kaloush, K. E., Faria, V. C., y Dos Santos Bardini, V. S. (2018). Performance characteristics offibermodified hotmixasphalt. Construction and Building Materials, 176, 747752. DOI: 10.1016/j.conbuildmat.2018.04.221

Koçkal, N. U., y Köfteci, S. (2016). Aggressive Environmental Effect on Polypropylene Fibre Reinforced Hot Mix Asphalt. Procedia Engineering, 161, 963-969. DOI: 10.1016 / j.proeng.2016.08.834

Li, Z., Zhang, X., Fa , C., Zhang, Y., Xiong, J., y Chen , $\mathrm{H}$. (2020). Investigation on characteristics and properties of bagasse fibers: Performances of asphalt mixtures with bagasse fibers. Construction and Building Materials, 248, 118648. DOI: 10.1016/j. conbuildmat.2020.118648

Liu, J., Li, Z., Chen, H., Guan, B., y Liu, K. (2020). Investigation of Cotton Straw Fibers for Asphalt Mixtures. Journal of Materials in Civil Engineering, 32(5), 04020105. DOI: 10.1061/ (ASCE)MT.1943-5533.0003181

Liu, Y., Zhang, Z., Tan, L., Xu, Y., Wang, C., Liu, P., Huayang, Y., y Oeser, M. (2020). Laboratory evaluation of emulsified asphalt reinforced with glass fiber treated with different methods. Journal of Cleaner Production, 274, 123116. DOI: 10.1016/j.jclepro.2020.123116

Lou, K., Xiao, P., Kang, A., Wu, Z., y Lu, P. (2020). Suitability of fiber lengths for hot mix asphalt with different nominal maximum aggregate size: A pilot experimental investigation. Materials, 13(17), 3685. DOI: 10.3390/MA13173685
Maharaj, R., Ali, R., Ramlochan, D., y Mohamed, N. (2019). Utilization of coir fibre as an asphalt modifier. Progress in Rubber, Plastics y Recycling Technology, 35(2), 59-74. DOI: $10.1177 / 1477760618795996$

Mansourian, A., Razmi, A., y Razavi, M. (2016). Evaluation of fracture resistance of warm mix asphalt containing jute fibers. Construction and Building Materials, 117, 37-46. DOI: 10.1016/j. conbuildmat.2016.04.128

Martinez Arguelles, G., Giustozzi, F., Crispino, M., y Flintsch, G. (2015). Laboratory investigation on mechanical performance of cold foamed bitumen mixes: Bitumen source, foaming additive, fiber-reinforcement and cement effect. Construction and Building Materials, 93, 241-248. DOI: 10.1016/j. conbuildmat.2015.05.116

Mawat, H. Q., y Ismael, M. Q. (2020). Assessment of moisture susceptibility for asphalt mixtures modified by carbon fibers. Civil Engineering Journal, 6(2), 304-317. DOI: 10.28991/cej-202003091472

Meng, F., Gao, D., Chen, F., y Huang, C. (2020). Fatigue Performance Test and Life Calculation of Fiber-Reinforced Asphalt Concrete. Annales de Chimie - Science des Matériaux, 44(2), 133-139. DOI: $10.18280 /$ acsm.440209

Miao, Y., Wang, T., y Wang, L. (2019). Influences of Interface Properties on the Performance of Fiber-Reinforced Asphalt Binder. Polymers, 11(3), 542-553. DOI: 10.3390/polym 11030542

Mohammed, M., Parry, T., Thom, N. H., y Grenfell, J. (2020). Microstructure and mechanical properties of fibre reinforced asphalt mixtures. Construction and Building Materials, 240, 117932. DOI: 10.1016/j. conbuildmat.2019.117932

Morea, F., y Zerbino, R. L. (2018). Improvement of asphalt mixture performance with glass macro-fibers. Construction and Building Materials, 164, 113-120. DOI: 10.1016/j. conbuildmat.2017.12.198 
Morova, N., Serin, S., Terzi, S., Saltan, M., Ozdemir Kucukcapraz, D., Sargin Karahancer, S., y Eriskin, E. (2016). Utility of polyparaphenylene terephtalamidefiberinhotmixasphaltasafiber. Construction and Building Materials, 107, 87-94. DOI: 10.1016 / j.conbuildmat.2015.12.193

Mrema, A. H., Noh, S. H., Kwon, O., y Lee, J. (2020). Performance of glass wool fibers in asphalt concrete mixtures. Materials, 13(21), 4699. DOI: $10.3390 / \mathrm{ma} 13214699$

Musa, S. S., Shanbara, H. K., y Dulaimi, A. (2020). The Effect of polypropylene fibres on the tensile performance of asphalt mixtures for road pavements. Materials Science and Engineering Conference Series, 888(1). DOI: 10.1088/1757$899 X / 888 / 1 / 012082$

Naseri Yalghouzaghaj, M., Sarkar, A., Hamedi, G. H., y Hayati, P. (2020). Effect of ceramic fibers on the thermal cracking of hot-mix asphalt. Journal of Materials in Civil Engineering, 32(11), 04020325. DOI: 10.1061/(ASCE)MT.1943-5533.0003396

Parimita, P. (2020). Influence of Natural Fibers as Additive on Characteristics of Stone Mastic Asphalt. IOP Conference Series: Materials Science and Engineering, 970(1), 012021. DOI: 10.1088/1757-899X/970/1/012021

Park, K., Shoukat, T., Yoo, P., y Lee, S.-h. (2020). Strengthening of hybrid glass fiber reinforced recycled hot-mixasphaltmixtures.Construction and Building Materials, 258, 118947. DOI:10.1016/j.conbuildmat.2020.118947

Perca Callomamani, L. A., Hashemian, L., y Sha, K. (2020). Laboratory Investigation of the Performance Evaluation of Fiber-Modified Asphalt Mixes in Cold Regions. Transportation Research Record, 2674(7), 323-335. DOI: $10.1177 / 0361198120922213$

Pirmohammad, S., y Hojjati, M. M. (2020). Influence of natural fibers on fracture strength of WMA (warm mix asphalt) concretes using a new fracture test specimen. Construction and Building Materials, 251, 118927. DOI: 10.1016/j. conbuildmat.2020.118927
Pirmohammad, S., Majd Shokorlou, Y., y Amani, B. (2020). Influence of natural fibers (kenaf and goat wool) on mixed mode I/II fracture strength of asphalt mixtures. Construction and Building Materials, 239, 117850. DOI: 10.1016/j. conbuildmat.2019.117850

Preciado, J., Martínez Arguelles , G., Dugarte, M., Bonicelli, A., Cantero, J., Vega, D., y Barros, Y. (2017). Improving Mechanical Properties of Hot Mix Asphalt Using Fibres and Polymers in Developing Countries. Materials Science and Engineering Conference Series, 245(2), 022013. DOI: 10.1088 / 1757-899X / 245/2/022013

Rahman, M. T., Mohajerani, A., y Giustozzi, F. (2020). Possible Recycling of Cigarette Butts as Fiber Modifier in Bitumen for Asphalt Concrete. Materials, 13(3), 734. DOI: 10.3390 / ma13030734

Ramalingam, S., Murugasan, R., y Nagabhushana, M. (2017). Laboratory performance evaluation of environmentally sustainable sisal fibre reinforced bituminous mixes. Construction and Building Materials, 148, 22-29. DOI: 10.1016/j. conbuildmat.2017.05.006

Romeo,E.,Freddi,F.,yMontepara,A.(2014).Mechanical behaviour of surface layer fibreglassreinforced flexible pavements. International Journal of Pavement Engineering, 15(2), 95-109. DOI: $10.1080 / 10298436.2013 .828838$

Sabaeei, A., Napiah, M., Sutanto, M., y Alaloul, W. (2019). Effects of Waste Denim Fibre (WDF) on the physical and rheological properties of bitumen. Materials Science and Engineering, Conference Series, 527(1), 012047. DOI: 10.1088/1757-899X/527/1/012047

Saleem, A. A., y Ismael, M. Q. (2020). Assessment resistance potential to moisture damage and rutting for HMA mixtures reinforced by steel fibers. Civil Engineering Journal (Iran), 6(9), 1726-1738. DOI: 10.28991/cej-2020-03091578 
Shanbara, H. K., Ruddock , F., y Atherton, W. (2018)b. A viscoplastic model for permanent deformation prediction of reinforced cold mix asphalt. Construction and Building Materials, 186, 287302. DOI:10.1016/j.conbuildmat.2018.07.127

Shanbara, H., Ruddock, F., y Atherton, W. (2018) c. A laboratory study of high-performance cold mix asphalt mixtures reinforced with natural and synthetic fibres. Construction and Building Materials, 172, 166-175. DOI:10.1016/j. conbuildmat.2018.03.252

Shanbara, H., Ruddock, F., y Atherton, W. (2018)a. Predicting the rutting behaviour of natural fibre-reinforced cold mix asphalt using the finite element method. Construction and Building Materials, 167, 907-917. DOI:10.1016/j. conbuildmat.2018.02.072

Sheng, Y., Zhang, B., Yan, Y., Li, H., Chen, Z., y Chen, $H$. (2019). Laboratory Investigation on the Use of Bamboo Fiber in Asphalt Mixtures for Enhanced Performance. Arabian Journal for Science and Engineering, 44(5), 4629-4638. DOI: $10.1007 / s 13369-018-3490-x$

Shubbar, A., Shanbara, H. K., Ruddock, F., y Atherton, W. (2020). Characterizing the Rutting Behaviour of Reinforced Cold Mix Asphalt with Natural and Synthetic Fibres Using Finite Element Analysis. Lecture Notes in Civil Engineering, 38, 221-227. DOI: 10.1007/978-981-13-7615-3_20

Souliman, M., Tripathi, A., y Isied, M. (2019). Mechanistic analysis and economic benefits of fiber-reinforced asphalt mixtures. Journal of Materials in Civil Engineering, 31(8), 04019142. DOI: 10.1061/(ASCE)MT.1943-5533.0002755

Takaikaew, T., Tepsriha, P., Horpibulsuk, S., Hoy, M., Kaloush,K.E.,yArulrajah,A.(2018).Performance of fiber-reinforced asphalt concretes with various asphalt binders in Thailand. Journal of Materials in Civil Engineering, 30(8), 04018193. DOI: 10.1061/(ASCE)MT.1943-5533.0002433

Usman, N., Masirin, M. I., Ahmad, K. A., y Ali, A. S. (2019). Application of recycled polyethylene terephthalate fiber in asphaltic mix for fatigue life improvement. Lecture Notes in Civil Engineering, 9, 1401-1413. DOI: 10.1007/978981-10-8016-6_101
Wang, H., Yang, Z., Zhan, S., Ding, L., y Jin, K. (2018). Fatigue Performance and Model of Polyacrylonitrile Fiber Reinforced Asphalt Mixture. Applied Sciences (Switzerland), 8(10), 1818. DOI: 10.3390/app8101818

Wang, X., Zhou, H., Hu, X., Shen, S., y Dong, B. (2021). Investigation of the Performance of Ceramic Fiber Modified Asphalt Mixture. Advances in Civil Engineering, 2021, 8833468. DOI: $10.1155 / 2021 / 8833468$

Wiśniewski, D., Słowik, M., Kempa, J., Lewandowska, A., y Malinowska, J. (2020). Assessment of Impact of Aramid Fibre Addition on the Mechanical Properties of Selected Asphalt Mixtures. Materials, 13(15), 3302. DOI: 10.3390/ ma13153302

Zhang, H., Hao, P., Pang, Y., y Mwanza , A. (2016). Design Method and Cost-Benefit Analysis of Hybrid Fiber Used in Asphalt Concrete. Advances in Materials Science and Engineering, 2016, 1-9. DOI: 10.1155/2016/8014704

Ziari, H., y Moniri, A. (2019). Laboratory evaluation of the effect of synthetic Polyolefin-glass fibers on performance properties of hot mix asphalt. Construction y Building Materials, 213, 459-468. DOI: 10.1016/j.conbuildmat.2019.04.084

Ziari, H., Saghafi, Y., Moniri, A., y Bahri, P. (2020). The effect of polyolefin-aramid fibers on performance of hot mix asphalt. Petroleum Science and Technology, 38(3), 170-176. DOI: 10.1080/10916466.2019.1697286

Los contenidos de este documento, representan insumos dentro de un proceso de gestión del conocimiento que, por sí mismos, a priori, no constituyen una declaración de una normativa, procedimiento, criterio o herramienta oficial de acatamiento obligatorio en la gestión de proyectos de obra vial pública de Costa Rica, por parte del LanammeUCR. Cualquier posición oficial para Costa Rica sobre aspectos puntuales contemplados en este documento, se realizarán por los medios que corresponden, según los lineamientos de la Universidad de Costa Rica, de la Ley 8114 y su Reglamento al Art 6 (Decreto 37016 - MOPT). 